\title{
EDIFICIOS PARA INSTITUTOS DE INVESTIGACIÓN DE LA UNIVERSIDAD DE SANTIAGO. SANTIAGO DE COMPOSTELA (GALICIA, ESPAÑA)
}

(BUILDINGS FOR RESEARCH INSTITUTES OF THE UNIVERSITY OF SANTIAGO, SANTIAGO DE
COMPOSTELA. GALICIA, SPAIN)
Fecha de recepción: 28-VIII-95

J.M. Gallego Jorreto, Dr. Arquitecto

\begin{abstract}
RESUMEN
En este año de 1995, cuando la Universidad de Santiago de Compostela celebra su V Centenario, han comenzado las obras de sus Institutos de Investigación. Se trata de cuatro edificios ubicados dentro del Campus Universitario que albergarán un Instituto de Investigación y un Instituto de Cerámica para la Universidad. Son Centros que, sin duda, servirán para potenciar la necesaria relación entre Universidad y Empresa. En el articulo se presentan las claves que permiten entender el diseño realizado y unos interesantes detalles constructivos del cerramiento de fachada que están en el origen de la propia apariencia arquitectónica que tendrán, en su conjunto, los edificios.
\end{abstract}

\section{SUMMARY}

In this year of 1995, when the University of Santiago de Compostela celebrates its 500th Anniversary, the construction works of its Research Institutes were started. These are four buildings located within the University Campus that will house a Research Institute and an Ceramics Institute for the University. These are centers which will undoubtedly serve to strengthen the required relations between the University and industries. The paper presents the key factors to permit a better understanding of the design and some interesting construction details of the enclosure walls are the basis of the architectural appearance of the future building complex.

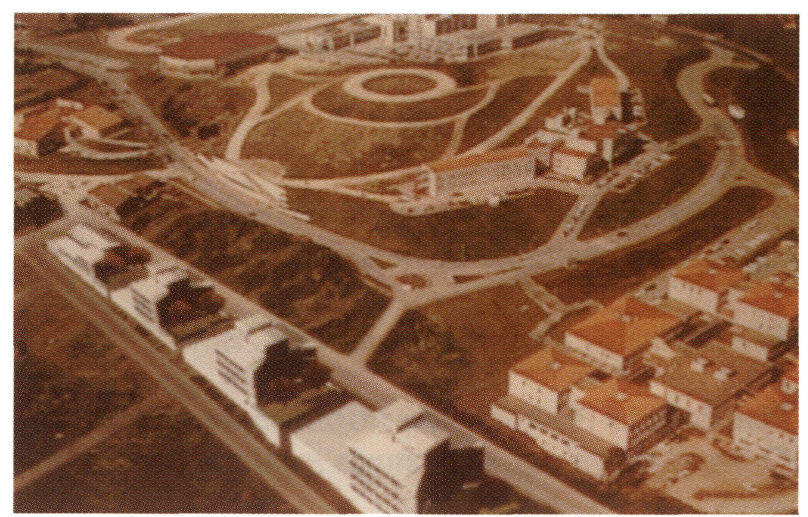

(c) Consejo Superior de Investigaciones Científicas Licencia Creative Commons 3.0 España (by-nc)

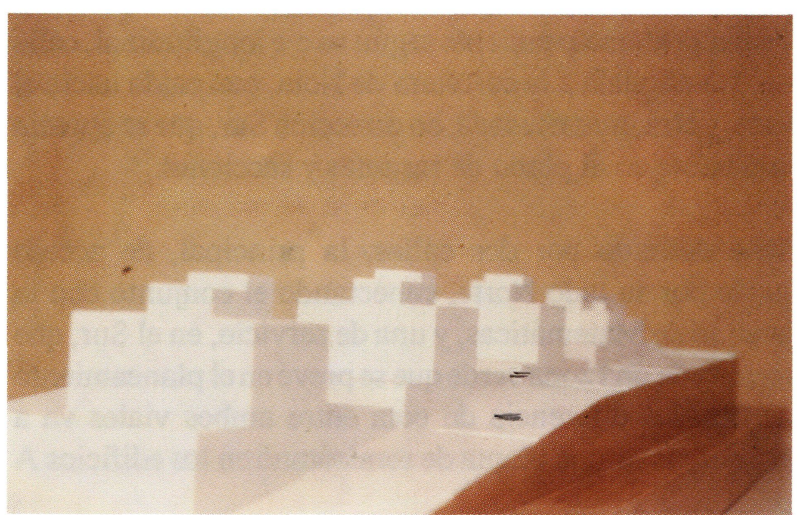

http://informesdelaconstruccion.revistas.csic.es 


\section{ENCARGO}

La Universidad de Santiago de Compostela, hace el encargo, en julio de 1993, de la Redacción del Proyecto de Ejecución de los edificios de Investigación del Campus. El encargo de la Redacción del Proyecto Básico de los tres edificios para Institutos de Investigación fue realizado el 9 de enero de 1992 y que unido al proyecto del Instituto de Cerámica, objeto de otro trabajo, constituyó un conjunto tratado como tal y, por tanto, con criterios de uniformidad. Así se contemplaba en el planeamiento existente en el campus de la Universidad.

Cumplido el primer requisito del encargo, que era la presentación de un Estudio Previo, que fue aceptado, se procedió a la redacción del proyecto básico, y con posterioridad, por encargo expreso de la Universidad de Santiago, la redacción final del Proyecto de Ejecución.

De acuerdo a lo que se decía en la Memoria del Proyecto Básico, se transcribe a continuación lo siguiente:

\section{EMPLAZAMIENTO}

Los edificios para Centros de Investigación se ubican en una franja de terreno colindante con la Facultad de Matemáticas, paralela a la carretera comarcal C-543, (avda. Mestre Mateo), que corresponde a la zona que el Plan Especial de Ordenación del Campus Universitario, PE-8, destina a Institutos de Investigación.

\section{SOLAR}

Dentro del espacio ya indicado, como continuación de la ordenación que éste inicia (el edificio del Instituto de Cerámica), se ocupa una parcela rectangular de $20 \mathrm{~m}$ de ancho y $171 \mathrm{~m}$ de largo, terminando en un triángulo de lados 20 y $22 \mathrm{~m}$, tal como se refleja en el plano de replanteo y topográfico.

El planotopográfico utilizado es el facilitado por la Universidad (Rigarpa S.L., ejecutado en agosto de 1991) que abarca el espacio de los edificios del proyecto y un área algo más extensa. Se ha incorporado al proyecto como Plano de Información.

El solar presenta pendiente según su eje longitudinal, calle prevista paralela a la carretera de Noia, con caída hacia el Oeste, y otra, más acusada, en dirección Sur, que se aprecia claramente en el plano de rasantes y secciones.

Viene definido por dos calles, la principal, de acceso rodado por su lado Norte, conectando el conjunto con la Facultad de Matemáticas, y una de servicio, en el Sur, que la separa de una zona verde que se prevé en el planeamiento previsto. La diferencia de cota entre ambos viales va a permitir ganar una planta de semisótano en los edificios A y $B$, y dos plantas en el edificio $C$, todas ellas hacia la vía de servicio.

Las rasantes que se han tomado son las deducidas del Plan Especial citado y del Topográfico facilitado, son, por ello, aproximadas y requerirán para su precisión los datos del proyecto de Urbanización, al menos el de la vía principal.

\section{PLANEAMIENTO Vigente}

La ordenación que afecta a la zona, lógicamente, es la que corresponde, de manera general, a la señalada en el Plan General de Ordenación Municipal y, en concreto para la zona, será su Planeamiento específico que es el Plan Especial de Ordenación del Campus Universitarios PE-8.

Éste contempla una zona específica y determinada para la instalación de los edificios de Investigación, entre la denominada Facultad de Pedagoxía y la de Matemáticas, que se denomina Z.E.U.-22 con un uso Docentelnvestigación.

El citado Plan Especial para esta zona dice textualmente: Se establece un área de movimiento que podrá ser ocupada al $50 \%$ por las nuevas edificaciones. La edificación en la parcela estará compuesta por un minimo de tres y un máximo de cinco pabellones exentos. La separación mínima entre pabellones será de $26 \mathrm{~m}$. La separación entre pabellones será constante.

Será obligatoria la disposición de las edificaciones en las Áreas de edificación definidas en planos de ordenación con frente al viario rodado Norte, al viario peatonal Sur y al borde Oeste de Área de movimiento. La superficie máxima construida será de $5.600 \mathrm{~m}^{2}$.

La edificación no superará, en el punto más desfavorable, la altura máxima de $16 \mathrm{~m}$ y 4 plantas, medidas desde la rasante de la via rodada Norte a la que da frente. La linea de cornisa de los edificios será horizontal y la misma para todos ellos en los frentes a las vías públicas rodada y peatonal.

Uso caracteristico: Docente -Investigación y administración pública.

\section{ORGANIZACIÓN PROPUESTA}

\subsection{Consideraciones previas}

Los condicionantes del proyecto se pueden ordenar y agrupar en los apartados siguientes:

\section{a) El cumplimiento del Planeamiento vigente}

El Plan Especial PE-8 establece los principios generales de la Ordenación, en cuanto a alturas máximas permitidas, 
edificabilidad máxima y número de plantas. Fijando, asimismo, como carácter espacial del conjunto, el de edificios iguales, o mejor casi iguales, con carácter de aislados, pero donde la relación entre ellos y la secuencia que provocan da una visión unitaria del conjunto. Edificios aislados pero proyectados conjuntamente.

b) La funcionalidad de los edificios y un uso aún no precisado

Su uso: un Instituto de Investigación es sobradamente conocido para poder aproximar, por los responsables com- petentes, sus demandas básicas, en cuanto a carácter y a espacio. Así se ha hecho. Pero el no tener precisado un uso concreto le da un cierto carácter abierto y esto va a ser determinante para acentuar su flexibilidad y poner en evidencia, así, su faceta de contenedor funcional y tecnológico.

c) La importancia del lugar

Su ubicación: dentro del Campus Universitario, en cota alta, con amplia ladera hacia la carretera de Noia. Su visión conjunta y lejana en un itinerario rodado y su visión
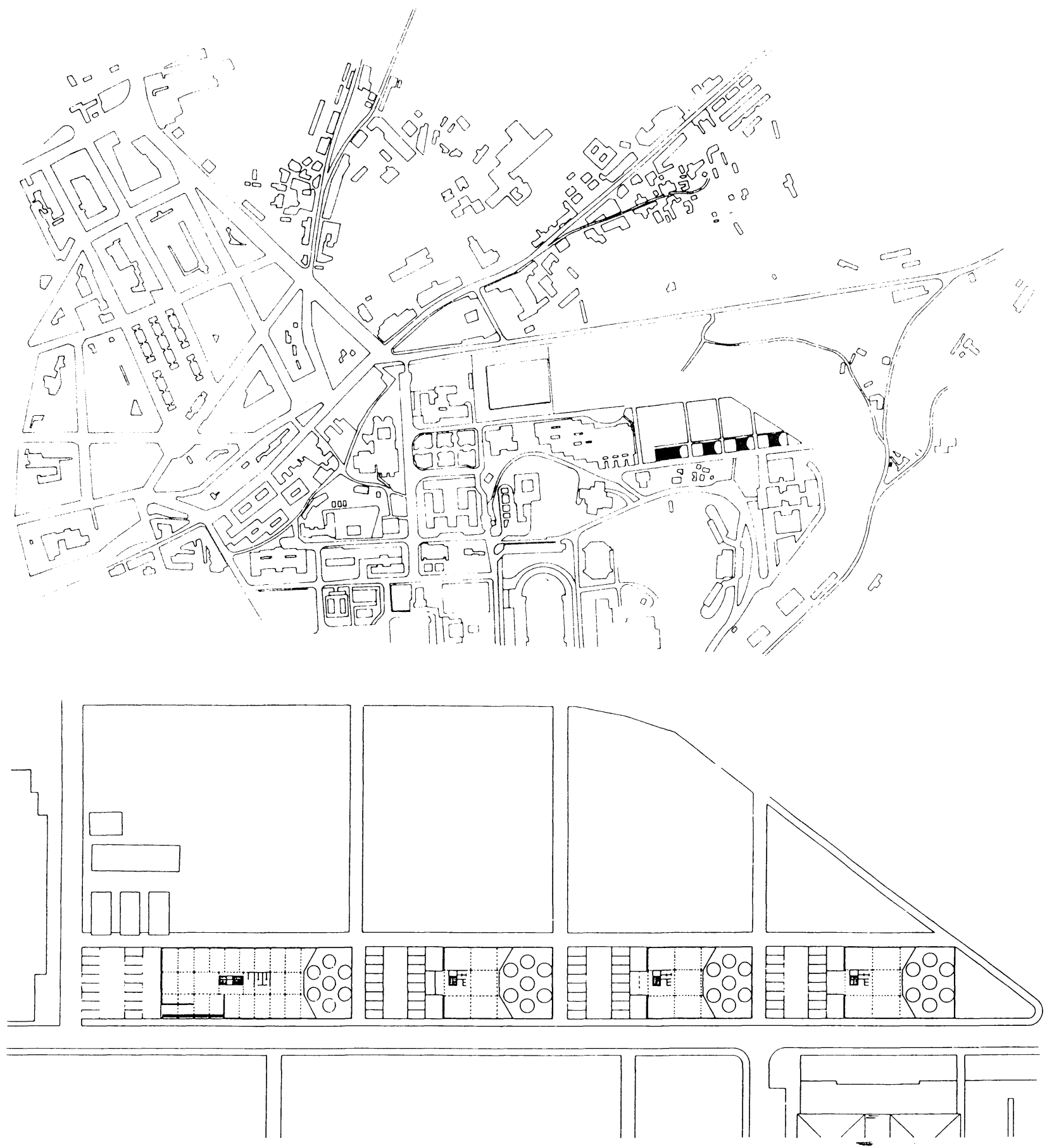


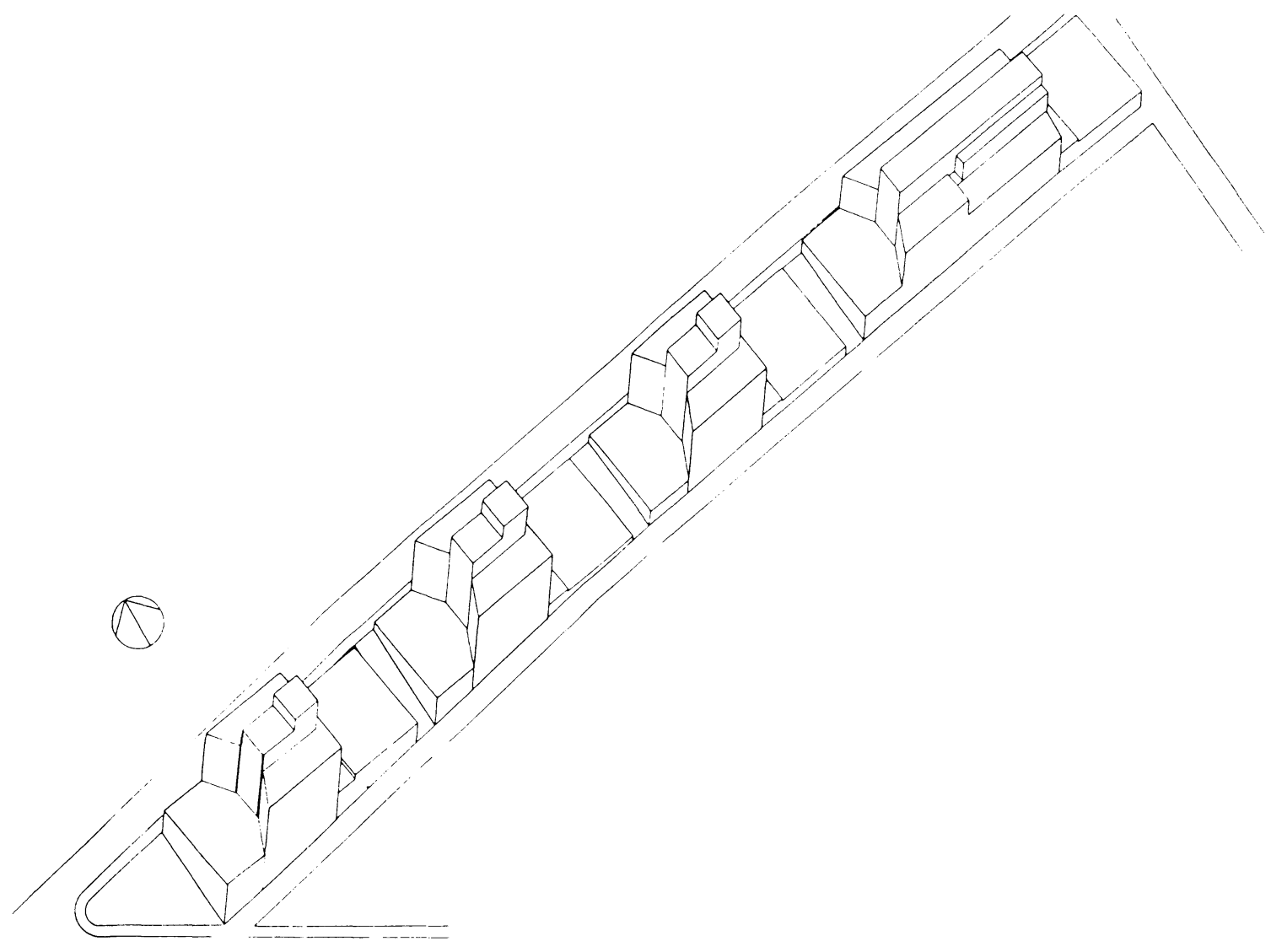

Axonométrica
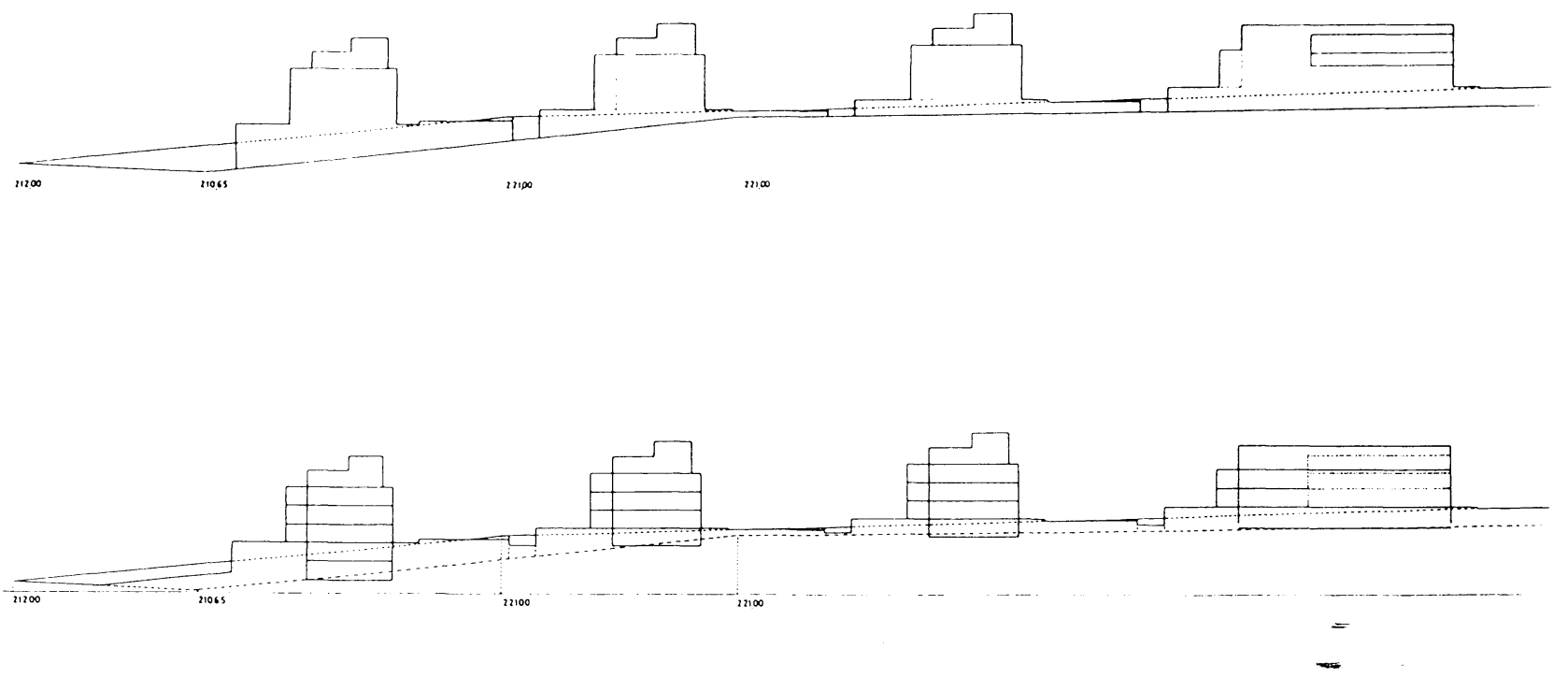

Ordenación. Alzado general.

(c) Consejo Superior de Investigaciones Científicas Licencia Creative Commons 3.0 España (by-nc) 
1 MUPO DE HORMGON UGERAMENTE ARMADO

2 GRANTO EXTRUDURO GRAVA FNA 2-5cm COLGADO JUNTA ABIERTA CONANCLANES DE ACERO INOX.TIPO AISI-304 (A-2) DE SUSTENTACIÓN ENLABASEY RETENCIÓNENCORONACIÓN

3 PROYECTADO DE $3 \mathrm{~cm}$ DE POUURETANO ( $30 \mathrm{~kg} / \mathrm{m}^{3}$ )

4 PLANCHA DE POUURETANO DE ALTA DENSIDAD

5 TABLERO APIMADO Y CANTEADO, LACADO EN BLANCO FOPMADO POR CALA DE CORTNERO

6 CORTNERO

7 ANGULAR 60X60 PARA ANCLAJE CARPINTERIA

8 GARRAS DE ANCLANE DE CARPINTERIA

9 CARPINTERIA DE AL LACADO COLOR GRIS PRACTICABLE PARA LIMPIEZA (BASCULANTE HACLA EL INTERIOR)O FRANCESA HACIA INTERIOA MERTE AGUAS Y RECOGIDA DE CONDENSACIONES)

10 FALSO TECHO ACUSTICO AMSTRONG (60X60)

11 CARRIL ELECTRIFICADO DE INSTALACIÓN DE FUERZA TIPO BUNNOOSBARRA 250 A. BLINDOJUNIO 125 A. Y $80 \mathrm{~A}$

12 VIERTEAGUAS DE CHAPA PLEGADA $(1 \mathrm{~mm}=0)$ DE AL. LACADO MISMO COLOR DE CARPINTERIA

13 CARRIL ELECTRIFICADO PARA ALUMBRADO, TELEFONIA, INFORMÁTICA Y PREVISIÓN OTROS USOS ELECTROPUNTO TIPO PLA-130- ELECTROSYSTEM

14 PAVMENTO DE PLAOUETAS DE (40X40) LOSETAS DE GRES PORCELANICO

15 VIERTEAGUAS SUPERIOR DE CHAPA PLEGADA ( $1 \mathrm{~mm}=e$ ) DE AL. LACADO VIERTEAGUAS DE CHAPA PLEGADA (1mm =)DE AL LACADO MISMO COLOR DE CARPINTERIA

16 PERSLANA TIPO WAMBI MOOELO PVC EN AL LACADO MISMO COLOR DE CARPINTERIA (CON LAMINA ORIENTABLE. REJA)

17 BARRAS DE ACERO ZINCADO (reja) DE PROTECCIÓN (CON RETENEDOR)

18 ENCIMERA DE TABLERO AGLOMERADO CON LAMINADO DE FORMICA ; alta presión Postforming)FRENTE CURVO

19 MURO DE BLOOUES DE GRANITO DE $15 \mathrm{~cm}$ Y CON ANCLANES SOLAMENTEA WUECO

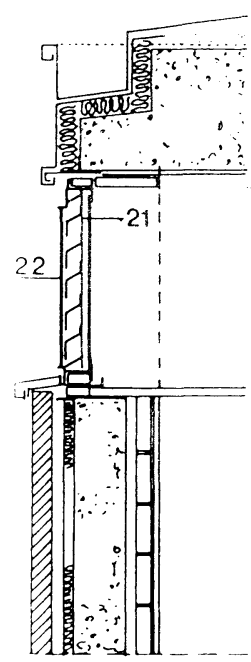

20 CUBIERTA DE ZINC ( junta alzada sobre fieltro

21 CANALON DE ZINC

22 VIERTEAGUAS DE ZINC

23 TUBO DE Fe 80X60 GALVANIZADO EN CALIENTE DE ANCLAJE DE CARPINTERIA ( anclado del Al.)

24 CARPINTERIA DE AL LACADO, CORREDERA

25 LAMAS TIPO LLAMBI PVC-100 DE AL. LACADO

( lama orientable standar)

26 VIERTE AGUAS DE CHAPA PLEGADA DE AL. $e=1,5 \mathrm{~mm}$

27 DOBLE LDE 90X90 DE Fe GALVANIZADO EN CALIENTE PARA SWECIÓN DECARPINTERLA

28 CORTINERO PERSLANA DE LAMAS ORIENTABLES ( TIPO GRADULUX DE LAMA FINA)

29 REVESTIMIENTO DE CHAPA DE AJ. LACADO CON AISLAMIENTO INCORPORADO

30 VENTANA DE A. LACADO PRACTICABLE SOLAMENTE PARA LIMPIEZA (BASCULANTE SUPERIOR)

31 VENTANA FIJA DE AL. LACADO

32 ENCIMERA DE TABLERO DM. $30 \mathrm{~mm}$ ANTIHUMEDAD, CANTEADO $Y$ LACADO

33 ESTANTERIA DE TABLERO DM ANTIHUMEDAD CANTEADOY LACADO

34 TABIQUES PALOMEROS de $8 \mathrm{~cm}$ CADA $50 \mathrm{~cm}$

35 TABLERO PREFABRICADO DE ARUTTA $\mathrm{e}=7 \mathrm{crm}$

36. CAPA DE COMPRESIÓN CON MALLA

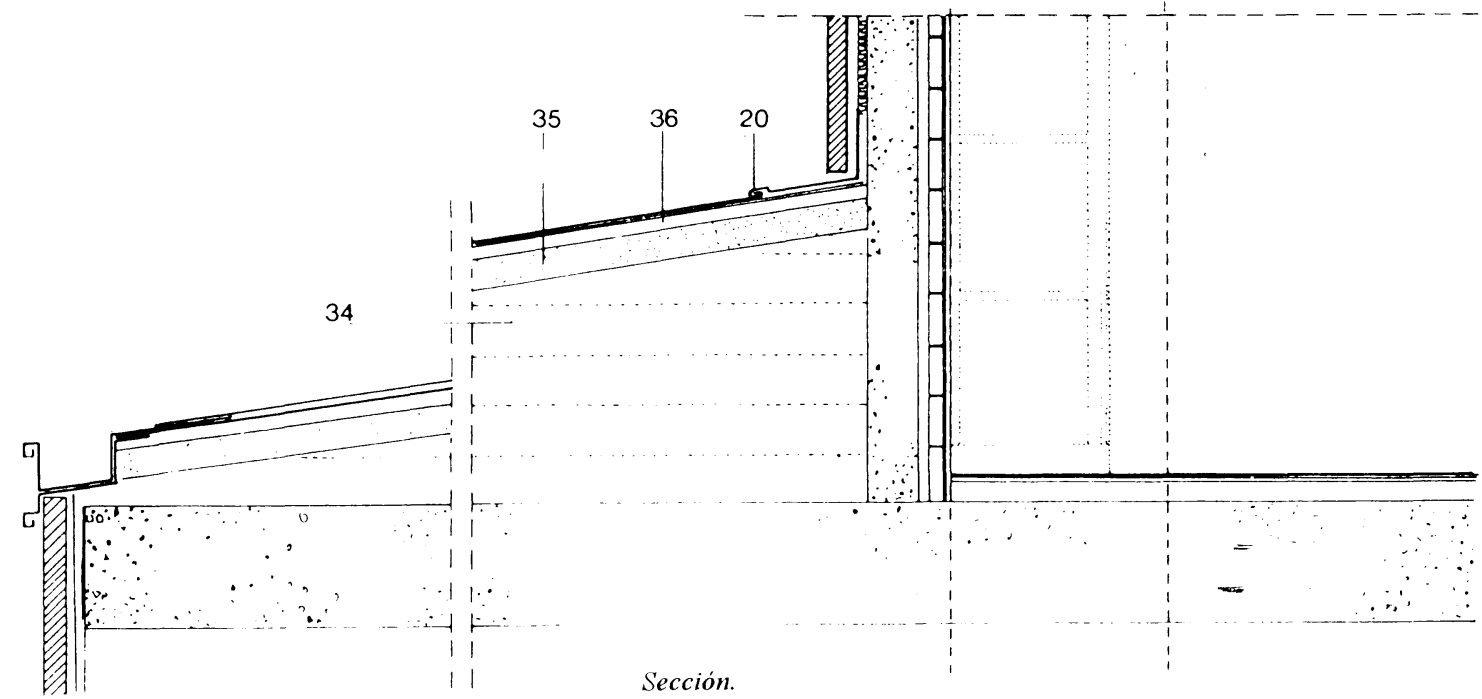

(c) Consejo Superior de Investigaciones Científicas 


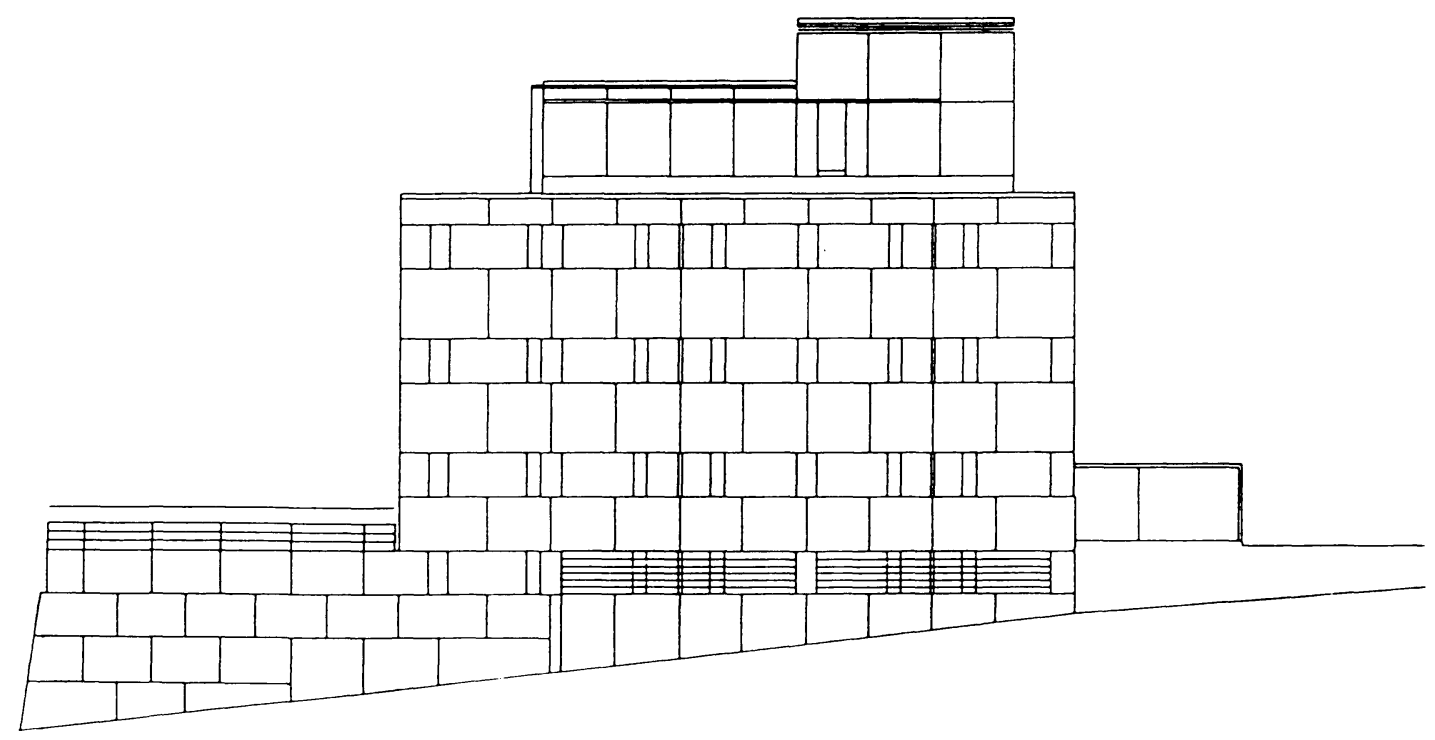

Alzado Sur (bloque B).

próxima desde la vía principal de acceso, deben ser factores contemplados en el proyecto, factores condicionantes de su resultado

\subsection{La solución propuesta}

La solución deberá dar una respuesta conjunta a los diversos problemas planteados.

En el proyecto del conjunto de los edificios se tiene en cuenta el lugar, con su doble interpretación: borde desde la parte superior, vía principal de acceso y balconada con vistas hacia el Sur y Oeste, y cornisa desde la zona inferior, la carretera de Noia.

Los edificios se plantean en conjunto, es decir, se presta atención a su valoración en serie o secuencial de elementos, atendiendo a su perfil, sus igualdades, semejanzas y su color. Su imagen sería de elementos pétreos en donde su secuencia repetida se matiza con las pequeñas diferencias y el color del granito.

Se proyectan tres edificios de iguales necesidades potenciales; las necesarias para un funcionamiento correcto. Las diferencias serán las que permite el ajuste concreto a su lugar determinado, la pendiente del terreno va a originar diferencias en sus semisótanos y jardines. Se va a ver acentuado el soporte de los edificios a modo de pedestal que va a matizar las diferencias entre ellos a la vez que refuerza, por su forma, el carácter direccional del conjunto, a modo de eje ordenador de esa zona concreta del Campus Universitario, subrayando el carácter institucional y representativo del conjunto.

Su silueta, su visión desde la cota inferior, se potencia con sus perímetros iguales y su aspecto pétreo y prismático; granito pulido, con diferencias de matiz en sus colores.

Cada edificio se proyecta, a su vez, con una cierta autonomía, la que exige y genera su propia organización. Ésta y su composición se fundamenta en el carácter principal de edificio de Investigación. (Laboratorios o no). Espacio para el estudio y el trabajo, con la posibilidad de la existencia de espacios recogidos y volcados a un espacio ajardinado (arbolado) propio del centro. Se crea un espacio que facilita la tranquilidad y la reflexión y que se integra a cada edificio, constituyendo parte de él. El arbolado denso le defenderá del sol del poniente.

El carácter tecnológico se centra en la búsqueda de las máximas posibilidades de espacios potenciales de laboratorios, zonas de estudio y trabajo. Espacios fácilmente relacionables donde los núcleos de escalera no los fraccione. Con servicios sanitarios y núcleo de comunicaciones verticales concentrado. Máxima diafanidad a través de la reducción del número de pilares Su tecnología se centra en un correcto sistema de iluminación y ventilación y la posibilidad de incorporar instalaciones o variarlas.

Para ello se proyecta un suelo técnico elevado y un falso techo acústico entre ambos, un espacio técnico capaz de acoger diversos tendidos, o incluso aire acondicionado si se desean.

El suelo continuo permite la variabilidad de las distribuciones que se plantean en posible mamparas móviles, en donde se incorporen instalaciones y se permita la conexión visual de los espacios a través de las convenientes zonas acristaladas. 

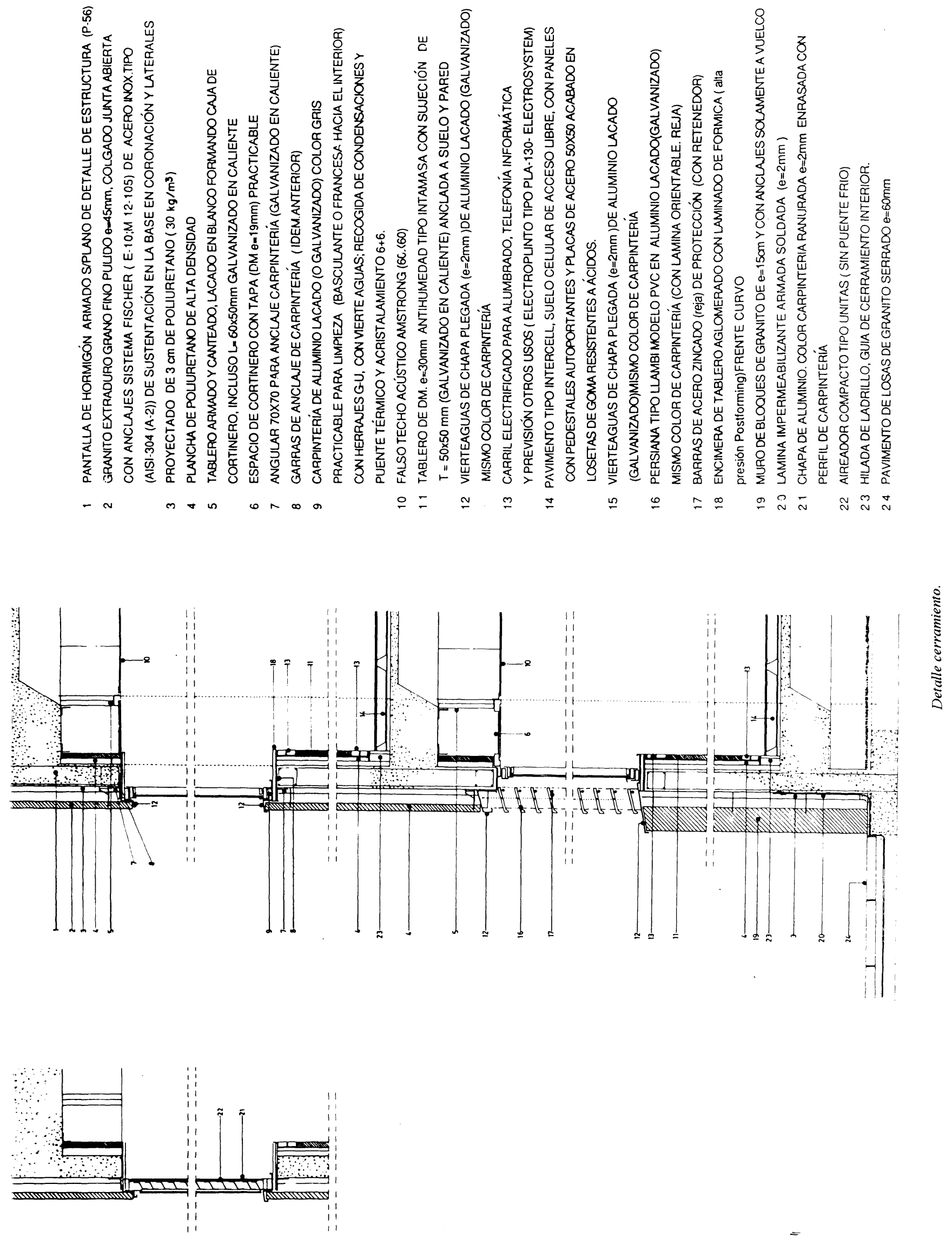


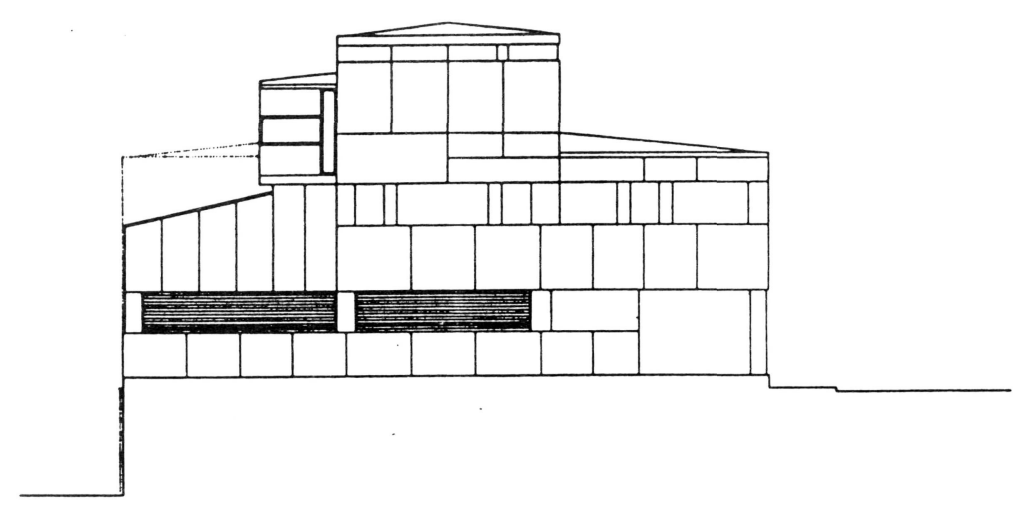

Alzado Este.

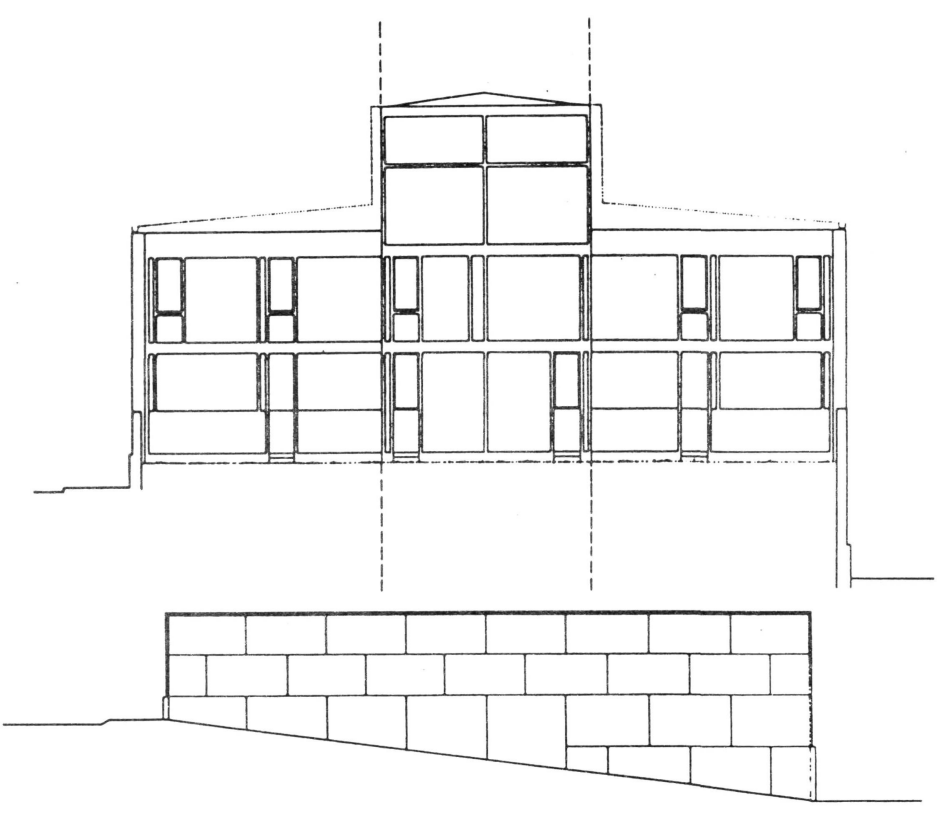

Alzado Oeste.

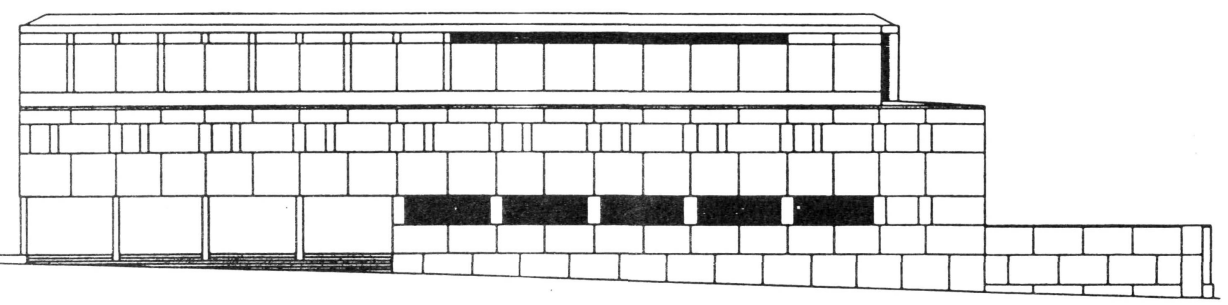

Alzado Norte.

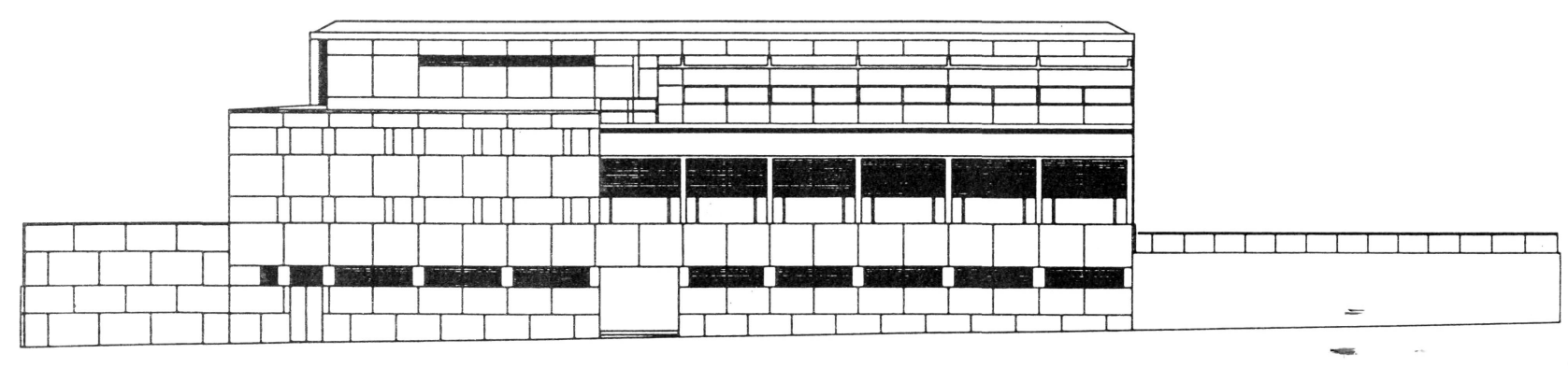

Alzado Sur.

(c) Consejo Superior de Investigaciones Científicas 


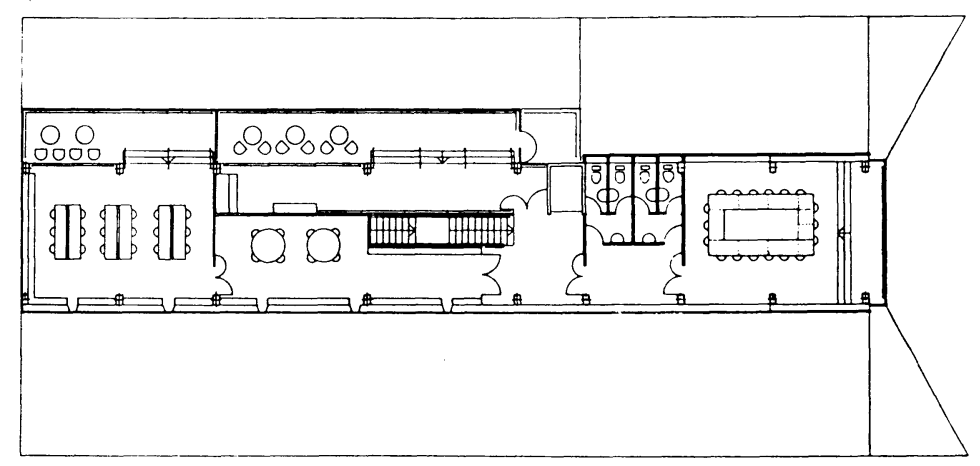

Planta $2^{a}$.

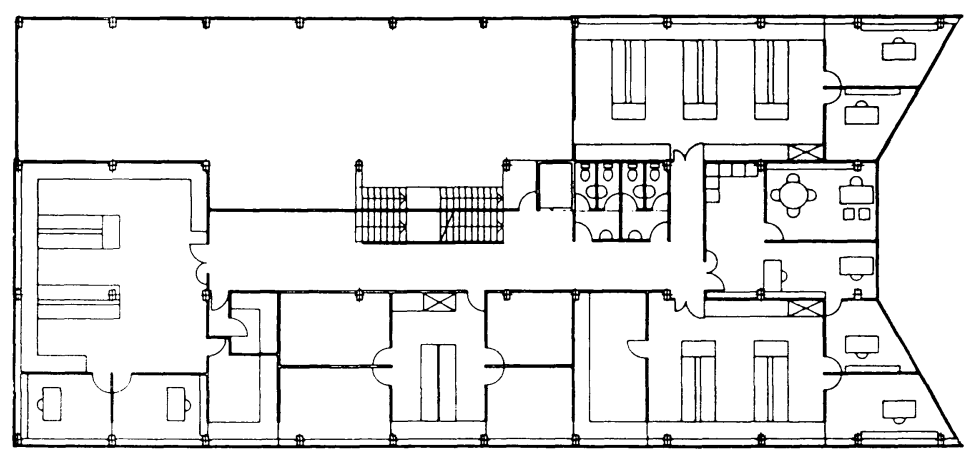

Planta $1^{a}$.

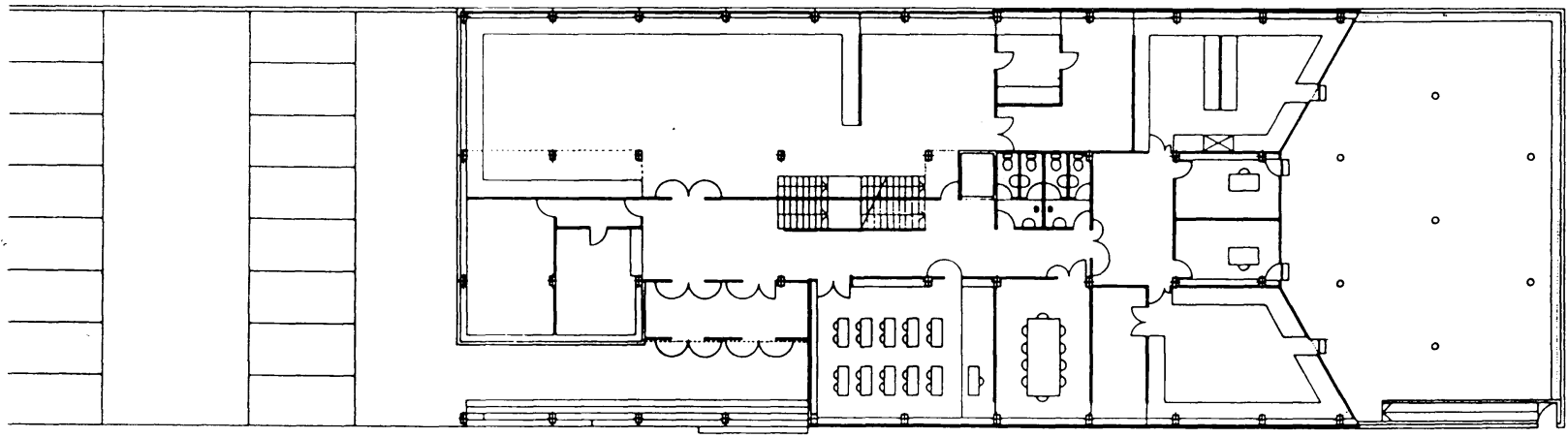

Planta baja.

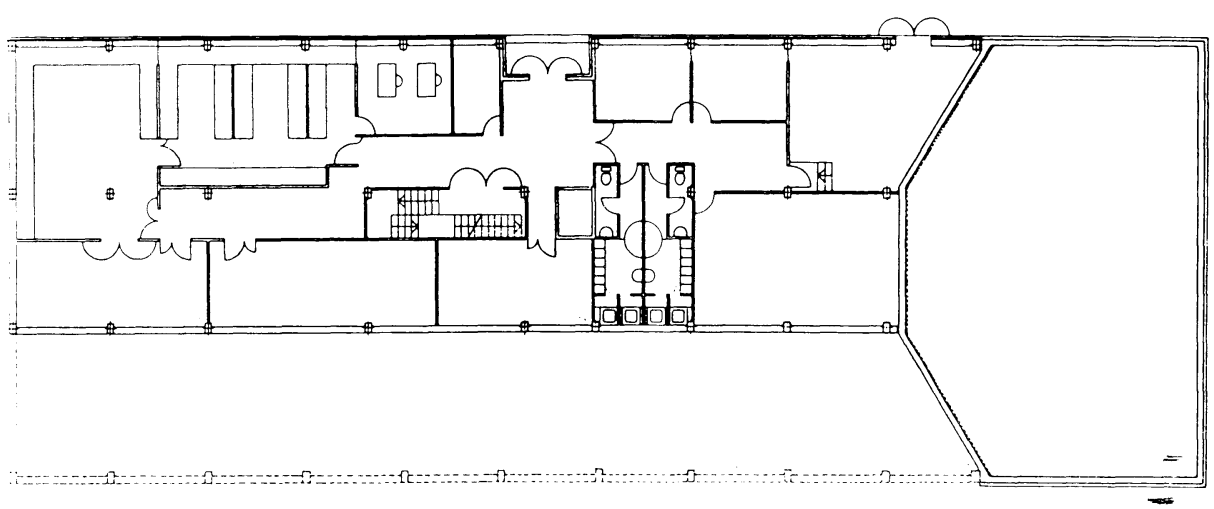


En el exterior, chapado de granito, intentando buscar la plástica de su uso más actual, al modo de grandes paneles colgados con junta abierta.

Las ventanas se plantean contemplando la posibilidad de su limpiezay ventilación, así comola de la salida al exterior de ventilaciones necesarias (aspiradores o ventiladores en laboratorios). El oscurecimiento y control solar a través del tipo de luna utilizada, de persianas ligeras interiores o de lamas orientables, con reja interior en planta baja para seguridad.

En la organización de los edificios se ha establecido un orden por plantas, orden lógico que dará rigor a la forma resultante, como argumento básico de su composición.

Planta Baja: acceso, acogida, conserjería, espacios administrativos, espacios de usos múltiples (clases, etc.) y espacios de reuniones o salas de juntas.

Planta Tipo: laboratorios y despachos. Espacios de trabajo y de investigación.
Planta última: espacio de biblioteca y estudio, volcado al jardín privado.

Se prevé una pequeña planta para posibles instalaciones (aire acondicionado, ascensores, etc.).

En los semisótanos, accesos de mercancías, archivos posibles o almacenes, instalaciones generales (calderas, calefacciones, grupos electrógenos), resto (laboratorios o zonas de trabajo).

La distribución y organización proyectada queda reflejada en los planos del proyecto. Se ha previsto su ajuste para el cumplimiento de la Normativa Vigente en todos sus aspectos.

En los diversos planos del Proyecto se presentan posibles variantes de uso que justifican su flexibilidad. Los detalles explican el proceso constructivo y dan idea de su aspecto acabado real.

\title{
publicación del IETCC/CSIC
}

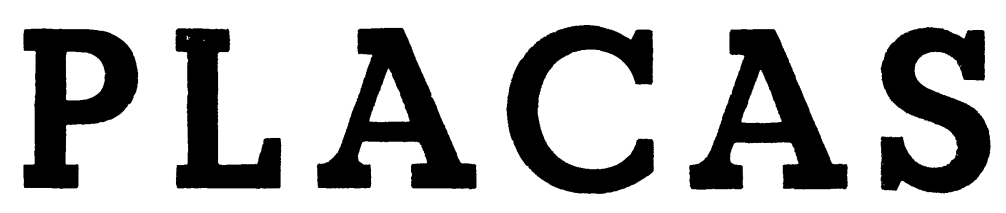

\author{
C. Stiglent gy H. Wippel \\ Drs. Ingenieros \\ Traducción de Juan Batanero \\ Dr. Ingeniero de Caminos \\ con la colaboración de \\ Francisco Morán \\ Ingeniero de Caminos
}

\begin{abstract}
Este libro, cuidadosa y magnificamente editado, reúne, quizás, la más completa colección conocida de tablas para placas, por los numerosos casos de vinculación y de carga estudiados y por la abundancia de relaciones de dimensión y de datos ofrecidos, que cubren prácticamente todo el campo de las losas en edificación. Permite desarrollar, con comodidad, rapidez y una aproximación suficiente, los cálculos de dimensionamiento y comprobación, obviando las dificultades que como es sabido, presenta el desarrollo numérico de los métodos de cálculo de estos elementos, evitando enojosas operaciones.

Trata la obra sobre "Zonas de Placas", "Placas sobre apoyos puntuales", "Placas apoyadas en dos, tres y cuatro bordes" y "Placas apoyadas elásticamente", tipos que en la actualidad disponian de una documentación, incompleta o nula, para la determinación de esfuerzos. Los corrimientos de la placa, como valores previos para la determinación de los momentos, han sido obtenidos por medio del Cálculo de Diferencias, método que se ha comprobado como suficientemente satisfactorio, aún en su forma simple, aplicado con un cierto control.
\end{abstract}

Un volumen encuadernado en tela, de $30,5 \times 23,5 \mathrm{~cm}$, compuesto de 92 págs. Madrid, 1968.

Precios: España, 2.500 ptas.; extranjero, $\$ 36.00$. 